\title{
KALMAN FILTER DESIGN FOR REAL-TIME ESTIMATION OF MODAL PARAMETERS AND CUTTING FORCE COEFFICIENTS FOR DETERMINING THE DYNAMIC STABILITY OF HIGH-SPEED LOW- RADIAL-IMMERSION MILLING PROCESSES
}

\author{
Josiah A. Bryan \\ Dr. Roger Fales, Dissertation Supervisor
}

\begin{abstract}
Chatter is a particularly severe and damaging form of tool vibration that can occur in milling processes under certain combinations of spindle speed and axial depth of cut. The ability to predict the onset of chatter in a particular tool-workpiece dynamic system could enable the development of a real-time control system that predicts and avoids chatter conditions. Various Extended Kalman Filters (EKFs) are considered here for estimating modal parameters or cutting force coefficients of the tool-workpiece dynamic system from tool deflection measurements in high-speed low-radial-immersion milling. These parameter estimates could be used to predict the dynamic stability of the system.

A novel application of an EKF is proposed here to estimate both a radial and a tangential cutting force coefficient. Separate sets of force coefficients for each cutting tooth may also be estimated, and the system is found to be observable. The EKF is tested on simulated measurements and found to perform accurately and repeatably. The EKF is also tested on experimental measurements, and the resulting parameter estimates result in simulations that correlate well with experimental tool deflection trajectories. Model modifications are also proposed that could improve accuracy, including the addition of centrifugal and Coriolis inertial forces and a means of accounting for tool eccentricity.
\end{abstract}

